\title{
Desarrollo de habilidades cognitivo lingüísticas (describir, resumir, explicar) empleando una metodología de resolución de problemas
}

\author{
Development of cognitive-linguistics abilities (to describe, to resume, to explain) based \\ in a problem resolution pedagogic model
}

Fredy Martínez Toro y Jaime Parra Martínez

Licenciados en Química Universidad Distrital Francisco José de Caldas. almatoazul@yahoo.com.mx, jaime.ep@gmail.com

\section{Resumen}

Este es el reporte final de una investigación realizada con diferentes grupos de educandos del nivel media básica en una institución del distrito. El enfoque principal fue el desarrollo de habilidades cognitivo-lingüísticas desde una visión socioconstructivista mediante el empleo del modelo de resolución de problemas. Se evidenció el desarrollo de las habilidades resumir, describir y explicar en el transcurso de las dases de ciencias, recolectando las actividades escritas que se les planteaban a los estudiantes; a partir de este material se realizó un análisis de carácter hermenéutico, el aul muestra un avance significativo en las habilidades de resumir, describir y explicar a la vez que se fomentaba la alfabetización científica.

\section{Palabras claves}

Habilidades cognitivo-lingüísticas, resolución de problemas, unidad didáctica, alfabetización cientúfica

\section{Abstract}

A final report from research in differents basic and media levels students groups in a district college. To development cognitive-linguistics skills and abilities from a socio-constuctivist sight was the main view through problem resolution pedagogic model. A best handling of cognitive-linguistics abilities to describe, to explain and to resume was evidenced in students work at the science class, recollecting write activities done for them; from this material by hermeneutic analysis, a significative advance in summaries, descriptions and explanations was demonstrated, at the same time, the scientific alphabetization was promoted.

\section{Keywords}

Cognitive-linguistics abilities, problem resolution pedagogic model, didactic unit, scientific alphabetization.

\section{Introducción}

La necesidad de implementar metodologías apropiadas para el desarrollo integral de los educandos en el aula de dase ha promovido todo un movimiento en procura de buscar nuevas y eficientes metodologías para alcanzar este fin. Dentro de este movimiento se puede encontrar el desarrollo de las habilidades cognitivo-lingüísticas; el cual se esta comenzando a implementar con la finalidad de superar aquellos problemas de comprensión y análisis que presentan los educandos, y la repercusión que esto tiene en el desarrollo del aprendizaje de las ciencias; promoviendo la educación como una actividad social y socializadora (Perales y Cañal, 2000) que emplea la 
comunicación como elemento fundamental para desarrollarse.

Durante la segunda mitad del siglo XX, la didáctica de las ciencias comenzó a adquirir cada vez mas fuerza entre educadores e investigadores del área de ciencias sociales enfocados en procesos educativos, a partir de esto han surgido un sinnúmero de publicaciones en las cuales se exponen nuevas metodologías para organizar el trabajo de aula; entre ellas el desarrollo de habilidades de carácter cognitivo y cognoscitivo (Garća, J; 1998; Jorba Et al., 1998,); estas características han surgido a partir de la evolución de los modelos que se han desarrollado durante los procesos de enseñanza aprendizaje en el último siglo.

La didáctica de las ciencias naturales es una disciplina que continuamente se reforma gracias a la búsqueda de mejores estrategias que desarrollen el aprendizaje de estas ciencias en los educandos, sin embargo, es construida a partir de cómo se observa el fenómeno del aprendizaje desde varias ciencias como la psicología, la sociología, la pedagogía, la historia y la epistemología de las ciencias que ofrecen una perspectiva que en conjunto modelan a partir de causas y procesos una interpretación aproximada a dicho fenómeno.

La visión de la ciencia desde el constructivismo es un aporte del desarrollo epistemológico de las ciencias que ofrece ventajas en la construcción del conocimiento de los educandos ya que parte del análisis de fenómenos basados en concepciones previas, generando los que se definen como problemas, fundamentados en un marco teórico extenso; en la presente investigación, se pueden definir como la realidad que rompe con todos los esquemas explicativos del individuo sumado a un interés por comprender la nueva situación.

La implementación de las situaciones problemas llevan a la creación o adopción de nuevos conceptos que permitan hacer interpretable la situación, posteriormente a una comprensión de la misma que en un proceso rećproco transforma las capacidades de inferir y analizar nuevas situaciones y redefinir los esquemas explicativos que se usaban hasta entonces.

Estas capacidades son las que evidenciamos en nuestro trabajo a partir de las llamadas habilidades cognitivo-lingüísticas (resumir, describir y explicar) que nos permiten entender la importancia del lenguaje en la interpretación de las situaciones problemas, teniendo en cuenta el contexto social y cultural del estudiante como su base para la construcción de conocimiento científico escolar, y como la óptica científica puede modificar su percepción del mundo permitiéndonos mostrar la significatividad que adquiere la interpretación de los fenómenos para los estudiantes.

Desde las teońas socio-culturales constructivistas del aprendizaje se destaca el papel central que tiene la comunicación en el proceso de enseñanza-aprendizaje, porque permite la negociación que conduce a pactos (Figura 1) (Perales y Cañal, 2000); siendo por lo tanto necesario considerar el uso de la lengua en la situación de aprendizaje como un factor determinante del aprendizaje significativo de los alumnos (Jorba et al., 1998). 


\section{TEORÍA SOCIOCULTURAL Y CONSTRUCTIVISTA \\ DEL APRENDIZAJE}

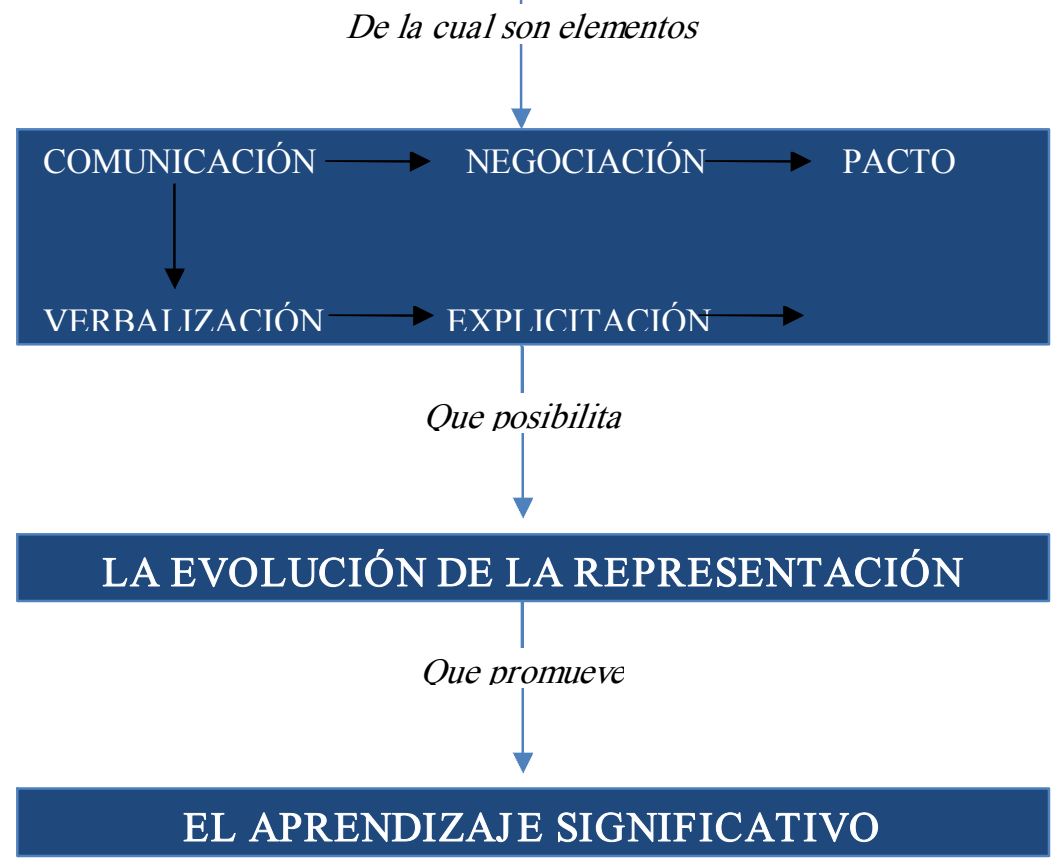

Figura 1. Marco teórico en el que se inscribe el trabajo de la lengua en situación de aprendizaje desde las áreas Curriculares Tomado de Jorba, Gómez, Prat; 1998

Jorba et al. (1998) postula el proceso mediante el cual los estudiantes pueden alcanzar las habilidades de describir, resumir y analizar entre otras (Figura 2), y como se ven afectadas por procesos intrapsicológicos (personales) e interpsicológicos (sodales).

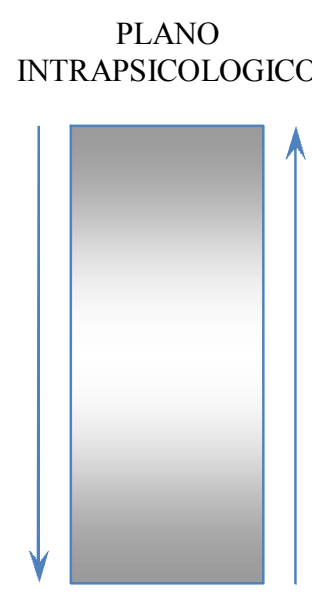

PLANO

INTERPSICOLOGICO
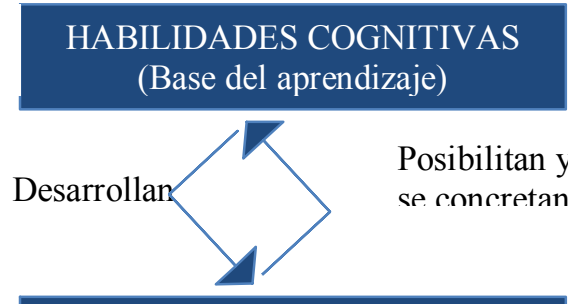

HABILIDADES

COGNITIVOLINGÜISTICAS

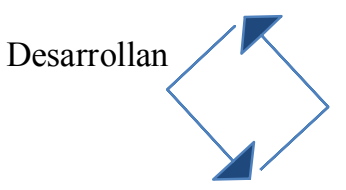

Las diferentes formas de usarlas determinan

maneras distintas

CONTENIDOS DE LAS ÁREAS CURRICULARES
MENTE

HUMANA

Posibilitan y se concretan

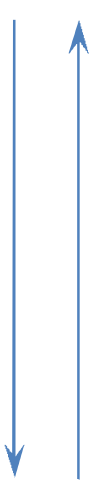

CULTURA

Figura 2 Visión sociocultural de la enseñanza-aprendizaje Tomado de Jorba, Gómez, Prat; 1998 
En el siguiente gráfico se definen algunas de las habilidades cognitivo-lingüísticas: describir, resumir y explicar que se analizaran en los estudiantes, las cuales se encuentran estrechamente relacionadas en los procesos cognitivos que se desarrollan en el aula de dase (Figura 3).

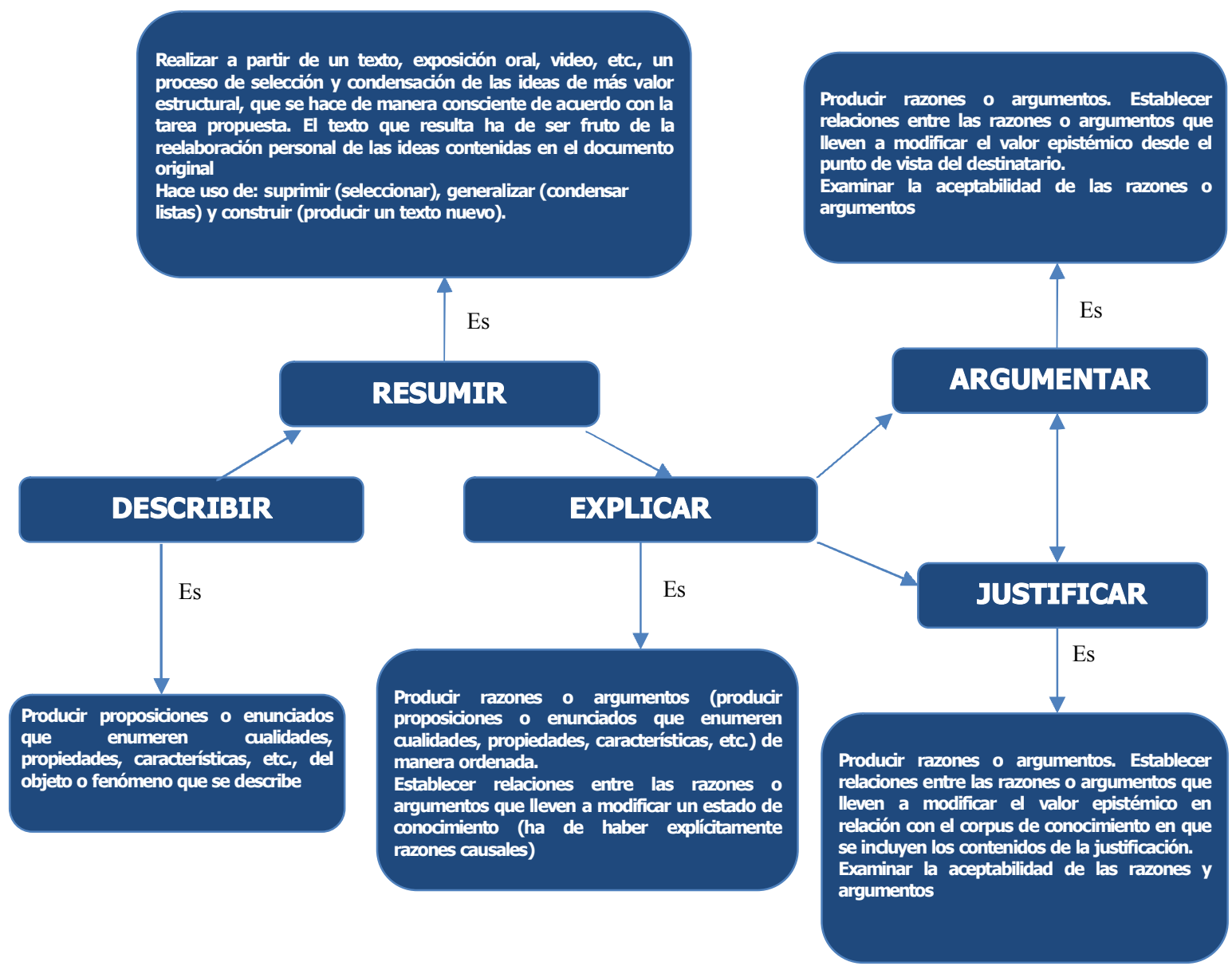

Figura 3. Habilidades cognitivolingüísticas Tomado de Jorba et al. 1998

La metodología que se aplico para el desarrollo de las habilidades cognitivo - lingüísticas en el transcurso del año se divide en dos: la aplicación de problemas, durante el primer semestre del año y la resolución de problemas de aplicación y ejercicios de reconocimiento durante el segundo semestre.

Uno de los factores mas importantes para iniciar el proceso de aprendizaje en la aplicación del modelo resolución de problemas es la motivación que despierte la situación problemática en los estudiantes, debido a la variedad de intereses que se pueden ver involucados en el aprendizaje de las ciencias, es bastante complejo estructurar una situación que induya la gran parte de los intereses que abarquen la motivación en los estudiantes, ya que requerińa la influencia del tema en todos los contextos, característica que depende de los conceptos que definen el tema, ya que aplicados en un contexto distinto en el que fueron diseñados, su significado puede ser mal interpretado por parte de los estudiantes.

Una de las ideas para la aplicación de los problemas no es concentrar el tema desde distintos enfoques sino desde contextos distintos desde donde se hayan realizado aportes a los conceptos; ej: los modelos atómicos pueden ser trabajados por problemas planteados a partir de los distintos contextos socioculturales sobre los que se han construido los distintos modelos, la motivación 
puede partir de la variedad de problemas en distintos contextos para ofrecer la ilusión de la elección en los estudiantes, ofreciendo distintas perspectivas de alguna agrupación de conceptos desde distintos contextos ya sean a través del tiempo, a niveles socioculturales, a niveles económicos distintos que sean capaces de captar la atención de los estudiantes sobre el tema de una manera tan sutil que se den cuenta una vez ya involucrados en su solución.

Los problemas que se emplearan son los que según Gil (Citado por García, J., 1998) se dasifican como ejercicios de reconocimiento y problemas de aplicación; se hace uso de este tipo de problemas debido a que las temáticas que se abordaron en el último período académico presentan un fundamento teórico bastante complejo, y extenso para que se pudieran abordar en su totalidad durante el ultimo período. Debido a esto el empleo de estos problemas y ejercicios permiten que los estudiantes se centren en lo que se busca abordar de las temáticas planteadas en el plan de estudios anual.

El objeto de nuestra investigación fue el desarrollo evolutivo de la capacidad de describir, resumir y explicar del estudiante. Este se abordo desde las unidades didácticas que se construyeron para los cuatro periodos; en estas se postularon trabajos de revisión bibliografica orientados por diferentes problemas (primer semestre), y problemas de aplicación (durante el segundo semestre).

Partiendo de los documentos escritos que producían los estudiantes se realizó un análisis de carácter hermenéutico para conocer los avances que el estudiante tenía. Se empleo este tipo de análisis debido a que se buscaba que el estudiante autorregulara este proceso evolutivo de las habilidades cognitivo-lingüísticas en la realización de sus escritos y mapas conceptuales en la interpretación de fenómenos desde las ciencias naturales, permitiéndoles actuar como reguladores de su proceso.

\section{Conclusiones}

Nuestra labor con los estudiantes se orienta en un enfoque pedagógico distinto al implementado habitualmente en la institución al pasar de un paradigma de enseñanza enfocado en el tradicionalismo, caracterizado por la asimilación de los conocimientos tal y como han sido formulados, el cual se presenta en un proceso de aprendizaje fundamentado en la descripción de los fenómenos y basada en la memorizadión (Sanmartí, N., Goméz, M., 1996), a un conocimiento orientado en la construcción personal en el aual se presta atención a las concepciones de los estudiantes, las cuales condicionan sus interpretaciones e influyen en el aprendizaje (Jiménez, M., 2000).

Los objetivos fundamentales de la resolución de problemas, es incentivar la curiosidad, interés, una postura critica y privilegiar el pensamiento divergente del estudiante (Liguori, L, Noste, I, 2005) y ponerlo en contacto con los fenómenos que se presentan a su alrededor; sin embargo la costumbre de trabajar a la luz de un modelo que los obligue a tomar un papel pasivo en el proceso de enseñanza aprendizaje hace que se cree una imagen de un mundo incomprensible y sin ningún tipo de aplicabilidad científica en la cotidianidad.

La ciencia al ser una construcción social para la interpretación de la realidad es realizada por medio del lenguaje permitiendo la comprensión por parte de los estudiantes del mundo en que viven. Buscando atender la diversidad contextual a la que se aplica la ciencia para captar la motivación de los estudiantes, este tema se abordó desde una variedad de enfoques problemáticos que posibilitaban a los estudiantes la facilidad de escoger y así decidir por donde comenzarían su camino al aprendizaje; no obstante el cambio de modelos en la enseñanza de la química fue bastante brusco tanto para estudiantes como para profesores, esto se manifiesta en la carencia de 
elección al determinar el enfoque del trabajo, ya que muchos estudiantes se les dificultó elegir en medio de las diferentes propuestas de trabajo.

En la introducción de la unidad didáctica de segundo periodo, se continúo con la implementación de múltiples problemas, dando tres tópicos principales de elección a los estudiantes, química de la naturaleza, química en la cocina y química en la industria; con la intención de enfocar a los estudiantes en un tópico bastante abierto con la posibilidad de elegir un problema. Se empleo un test; en este test se les pedía que plantearan un ejemplo en cada uno de los tópicos antes mencionados, las preguntas mas frecuentes fueron "ccomo extraer la esencia de una manzana?", "¿Cómo extraer el aroma de una naranja?" ó "¿Cómo extraer el aroma de una mandarina?" entre otros. Por otro lado, algunos no tenían la noción de formular una pregunta de manera coherente, 0 que fuera una pregunta a partir de una duda verdadera; a lo cual formulaban una pregunta que probablemente para ellos les parecía un problema pero que en realidad ya tenía solución desde la red cognitiva preestablecida; esto se comprobó auando se les hacia la pregunta que ellos mismos habían formulado, pero con una diferencia semántica.

Otro de los inconvenientes manejados en esta área fue la formulación de pasos para resolver el problema, determinando un orden que se asemeja a un método de construcción de ciencia escolar, ayyo orden, coherencia y organización determinaría la fortaleza de su investigación y la aplicación de está a la interpretación de la realidad; para lo cual era necesario que desarrollaran la capacidad de regular y organizar sus esfuerzos para que la investigación fuera más efectiva y coherente, capaz de asemejarse al conocimiento construido por la ciencia formal.

Dentro de lo observado en los trabajos de los estudiantes se encontró que la mayoría de consultas era una copia tomada de intemet 0 de otras fuentes sin que se evidenciara un cambio 0 una construcción personal en la misma; a partir de esto se generaron varías hipótesis sobre el hecho de emplear este tipo de "estrategias" de resolución del problema.

Uno de las dificultades determinantes en el trabajo de los estudiantes, fue el poco acceso a recursos que permitieran un desempeño más concreto sobre la realidad que trabajaban, muy pocos de los trabajos de los estudiantes tuvo una aplicación real o práctica, por lo cual las ideas desarrolladas, aunque buenas se quedaron en un plano del que fue difíil avanzar. Esto debido a factores logísticos de tiempo, espacio y materiales para la realización de estas prácticas, si la motivación de algunos estudiantes se encontraba en el desarrollo de experiencias en el laboratorio, esta se tomaba imposibilitante.

En la gran mayoría de los trabajos se evidenció la falta de lectura en la consulta de los estudiantes; refiriéndonos a una lectura comprensiva que les permitińa discernir y seleccionar la información relevante, expresar un punto de vista distinto al formulado, o comprender lo consultado; lo aral se veía reflejado en la falta de coherencia en la consulta y de lectoescritura en el trabajo.

El resultado de la implementación de esta unidad se determino por la cantidad de estudiantes que desarrollaron el trabajo de una manera honesta y responsable; convergiendo en una construcción personal sobre las temáticas abordadas relacionadas con su cotidianidad. Se evidenciaron falencias en la valoración de los trabajos de los estudiantes, en donde la solución al problema fue muy básica; en otros, donde existía un conglomerado de conceptos sin coherencia que dificultaba la labor del lector; y por último, en la cantidad de estudiantes en donde los procesos de motivación desarrollados anteriormente tanscurrieron sin resultados visibles.

Este conjunto de reflexiones sumado al tema a enseñar en el segundo periodo, llevaron a un cambio metodológico de las unidades didácticas; ahora se buscaba llevar a los estudiantes a 
comprender información científica (complejidad progresiva) que les permitiera definir su realidad bajo preceptos científicos sencillos y un método propio de investigación científica escolar: Se continúo con el desarrollo en las habilidades cognitivo-lingüísticas, realizando actividades orientadas a mejorar la descripción, redacción de resúmenes y explicación. Con lo cual se esperaba que llegasen a un nivel de construcción de un punto de vista propio distinto al de un texto de información en ciencias frente a una situación problema.

El trabajo desarrollado con los estudiantes fue diferente al de los periodos anteriores, ya consistía en determinar las ideas principales y secundarías de textos informativos cuyo fundamento científico era el mismo pero el enfoque y la estructura eran distintos, variando desde el más académico "El mundo de la química, Capitulo III: los elementos químicos", hasta uno más informal "¿Por qué somos de carbono y no de silicio?".

El trabajo se basaba en desarrollar una lectura comprensiva, que venía determinada por la extracción de ideas principales y secundarias del texto, los conceptos relevantes y la construcción de redes de ideas o "mapas conceptuales" para la comprensión del texto. En estos resultados, se ve un avance ya que se crea un método sencillo para comprender la macroestructura de un texto, y la información que guarda en él.

Finalmente los estudiantes al ir culminando el año lectivo mostraron una mejora en los procesos de lectoescritura, esto se evidenció en la facilidad de extraer ideas primarias por pánafos y de los conceptos fundamentales; esta comprensión nos arroja el uso de palabras desde el mismo contexto de la lectura, incluso hasta ideas, lo aual denota la dificultad a la hora de formular una idea comprendida en palabras propias, dejando un camino para resolver el análisis del texto: ver la coherencia de la idea principal expuesta por el estudiante en relación con la idea principal del texto, donde solo algunos llegaron a exponer coherente y acertadamente las ideas más relevantes del mismo.

El empleo de red de ideas o "mapas conceptuales" fue para algunos estudiantes un éxito, ya que en el manejo de esta herramienta partieron de cero y ahora solo hay que corregir algunos errores pero no con la confección del mapa sino con el tema en cuestión (parte conceptual).

Una de las dificultades de trabajar este tipo de metodologías es la sola relación textual, ya que por falta de tiempo no se logró concretar actividades de tipo narrativo que permitieran conceptualizar fenómenos y reforzar la parte descriptiva y explicativa de la ciencia, en un proceso oratorio.

\section{Bibliografía}

GARCIA J.J. (1998) Didáctica de las ciencias resolución de problemas y desarrollo de la creatividad., Ed. Colciencias-Facultad de Educación de la Universidad de Antioquia.

JIMÉNEZ, M., EN PERALES, Y CAÑAL (2000). Modelos didácticos. En Perales, F., Cañal, P., (2000). Didáctica de las ciencias experimentales (Cáp. 7). España: Editorial Marfil, S.A.

JORBA J., GOMEZ I., PRAT A. (1998) Hablar y escribir para aprender. Ed. Síntesis-España.

LIGUORI, L., NOSTE, I., (2005). Enseñar ciencias naturales. En Didáctica de las ciencias naturales, Argentina: Ediciones HomoSapiens

PERALES J., CAÑAL P. (2000) Didáctica de las ciencias experimentales: teoría y práctica de la enseñanza de las ciencias. Ed. Marfil-España

SANMARTÍ, N., GÓMEZ, M, (1996, Julio) La didáctica de las ciencias: una necesidad, Educación Química, 156 - 168. 